\title{
Interactive comment on "New particle formation from sulfuric acid and ammonia: nucleation and growth model based on thermodynamics derived from CLOUD measurements for a wide range of conditions” by Andreas Kürten
}

Anonymous Referee \#2

Received and published: 25 February 2019

Andreas Kürten presents the extension of his previous model for sulfuric acid - dimethylamine nucleation. The model is extended to include evaporation intended for modelling sulfuric acid - ammonia nucleation. The main goal of the work is to derive new thermochemical parameters from CLOUD data. Understanding the mechanisms of sulfuric acid nucleation is of general interest and the presented model provides a great addition to the toolbox.

The manuscript is well written and structured and I can recommend publication in Atmospheric Chemistry and Physics and the following minor comments have been ad- 
dressed.

Line 161: How computationally heavy are these simulations? It would be beneficial to indicate the runtime that a typical simulation takes.

Line 167-169: The maximum amount of ammonia molecules in the simulations are not allowed to exceed the number of acid molecules. How well is this assumption justified? Based on quantum chemical data (Olenius et al 2013, Elm et al 2017) on sulfuric acid ammonia clusters this assumption seems somewhat reasonable, but it might be worth at least checking that the omission of clusters with one more base molecule than acid molecule present is not a large source of errors.

Line 170-172: In the pentamer and larger clusters there is not differentiated regarding the amount of bases. How large a source of errors does this assumption lead to? Surely, the 5 sulfuric acid cluster without any bases or only 1-2 ammonia molecules present are not very stable.

Line 182-185: While the results would most likely not be drastically different, the simplification of the evaporation rates, put a very strict constrain on the nucleation mechanism. Can it be quantified how much this simplification influences the results?

Line 188-193: It seems like an odd choice to take some of the cluster thermodynamics from a different study and not fitting them like the remaining. Why was this choice made?

Line 408-411: It should be mentioned that coagulation can also be the cause of the curvature in the line.

Line 481-482: Looking at Table 1 it appears that the quantum chemical values taken from Ortega et al 2012, fits quite well with the estimated values using the optimization method. This is also evident from comparing Figure 2 with Figure S1. Perhaps it should be further emphasized that the optimization of the values is more or less redundant and that quantum chemically obtained entropy values are quite accurate. 
Interactive comment on Atmos. Chem. Phys. Discuss., https://doi.org/10.5194/acp-2018-1323, 2019.
ACPD

Interactive comment 